\title{
Automatic Ultrasound Image Analysis in Hashimoto's Disease
}

\author{
Robert Koprowski ${ }^{1}$, Zygmunt Wrobel ${ }^{1}$, and Witold Zieleznik ${ }^{2}$ \\ ${ }^{1}$ University Of Silesia, Faculty Of Computer Science And Materials Science, Institute \\ Of Computer Science, Department Of Biomedical Computer Systems, Ul, Bedzinska \\ 39, 41-200 Sosnowiec, \\ ${ }^{2}$ Internist's Office, ul. Dworcowa 25, 41-902 Bytom, Poland \\ koprow@us.edu.pl, wrobel@us.edu.pl, wzieleznik@gmail.com
}

\begin{abstract}
The paper presents diagnostics of parenchyma echogenicity and organ dimensions in thyroid examinations in the case of Hashimoto's disease using image processing methods. In the event of discovering focal changes within the thyroid, a method for their pathology evaluation was suggested. The detector proposed operates fully automatically; using the information on the image texture it detects an artery in the image, which fulfils the role of reference point, and based on it - detects the area of interest.
\end{abstract}

\section{Introduction}

The tissue of regular thyroid features homogeneity and high echogenicity, which determines follicular structure of the gland [4]. In autoimmunological inflammation the follicles architecture destruction and lymphocytic infiltrations occur, what is the reason of echogenicity decrease [5]. Till 2000 the change in echogenicity was described as a subjective parameter, which was evaluated based on rough visual comparison with the surrounding muscular tissue of the neck (most frequently with the sternocleidomastoid muscle) [6], [7]. Now a computer histogram of grey scale is suggested for quantitative measurement of echogenicity decline in the thyroid in autoimmunological inflammations [7], [8], [9], [10], [12], [13]. This method excludes the subjective element in echogenicity evaluation, what has a substantial impact on the value and repeatability of ultrasonographic examination [11]. The lack of procedure standardisation is a significant drawback of this method, because individual authors were using various initial settings of the ultrasonograph, what affects gland's echogenicity. The control group in this study consisted of 10 volunteers without clinical symptoms, without a thyroid disease and illnesses of immunological basis in anamneses, with proper results of anti-TPO, anti-TG antibodies level and with proper TSH, FT3, FT4 levels as well as 10 volunteers with Hashimoto's disease. Thyroid examinations were performed (in both groups) within a week starting from subjective and objective examinations up to laboratory investigations. For each patient one image of the left and one of the right side of the disc was obtained (Fig. 1). 

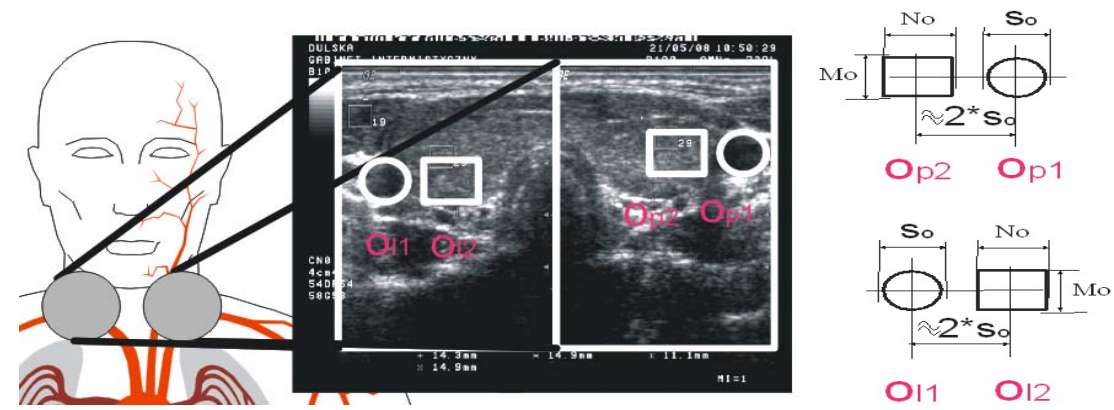

Fig. 1. Method of thyroid images obtaining and areas of $o_{l 1}$ and $o_{p 1}$ arteries, $s_{o}$ in diameter, and also determined based on them, using the described algorithm, areas on thyroid lobes $o_{l 2}$ and $o_{p 2}$ of $M_{o} x N_{o}$ dimensions

In the image presented in Fig. 1 , areas $o_{l 1}, o_{l 2}, o_{p 1}, o_{p 2}$ are marked, which will be automatically detected using the algorithm presented in this paper. Because of the image specific nature (Fig. 1), arteries on the left and right side will be initially detected, denoted as $o_{l 1}$ and $o_{p 1}, s_{o}$ in diameter and then, on their basis, the areas $o_{l 2}, o_{p 2}$ on thyroid lobes, of $M_{o} x N_{o}$ size.

From among known methods for texture analysis: statistical approach, structural method, transformation methods or model-based methods, a hybrid approach has been suggested, combining two aforementioned methods (statisticalstructural).

\section{Statistical-Structural Method}

\subsection{Image Preprocessing}

Image $L(m, n)$ in grey levels, where $m$ - line, $n$ - column, of $M x N=620 x 400$ resolution, is obtained from USG apparatus with a $9 \mathrm{MHz}$ head. Then the filtering operation is carried out using a median filter of mask size $M_{h} x N_{h}=3 x 3$ pixels [1], [2]. In the next stage the illumination unevenness is removed from the

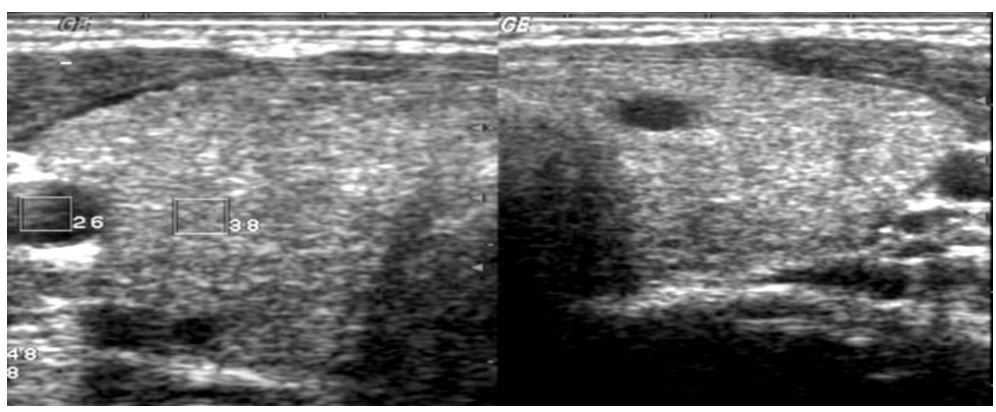

Fig. 2. Input image $L_{M}$ 


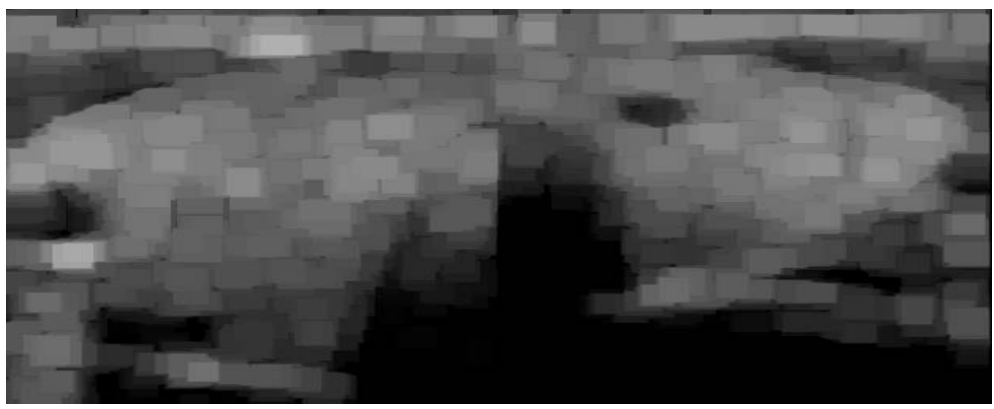

Fig. 3. Result $L_{0}$ of input image $L_{M}$ opening

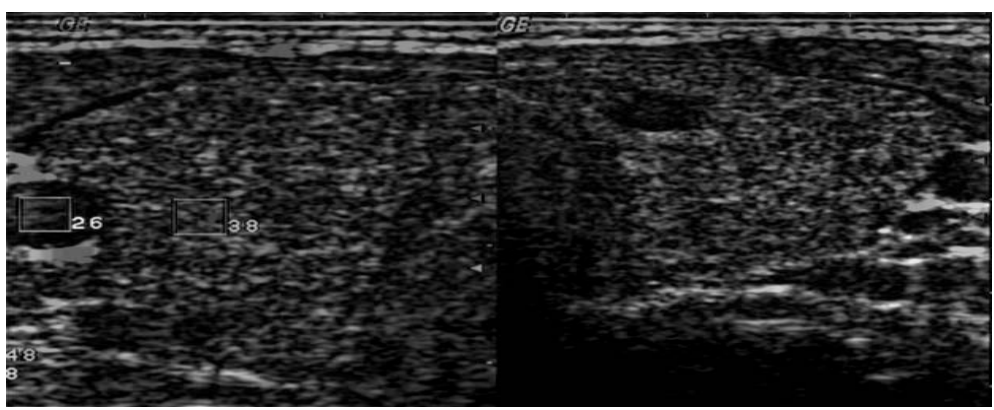

Fig. 4. Image $L_{T}$ as normalised $L_{M}-L_{0}$ difference

image, what results in partial absorption and dispersion of ultrasonic beam by individual organs (objects).

To this end the operation of morphological opening was carried out (image created $-L_{o}$ ) with a structural element $S E$ of $M_{S E} x N_{S E}=19 x 19$ pixels size. Image $L_{T}$ with removed background is computed as the difference $L_{M}-L_{o}$ normalised to $0-1$ interval. Image $L_{T}$ created this way is fed to objects texture detector input.

\subsection{Suggested Detector Description}

Image $L_{M}$ is then subject to operations of erosion (Fig. 5), obtaining image $L_{e \alpha}$, using structural element $S E 2$ of $M_{S E 2} \times N_{S E 2}=3 x 3 k$ pixels size for $k=1,2,, 16,17$. Each resultant image $L_{k}$ for $k=3,6,9,12,15,17$ has been shown in the form of a colour contour in image $L_{M}$ - Fig. 6 .

Resultant image $L_{k=17}$ has been further used to determine circles by means of Hough's transform. Groups of pixels of $L_{b}$ contour image of circle of radius $r_{0}=20,21,22,, 89,90$ have been sought. The circles radii interval was assumed based on anthropometric and anatomic data of arteries cross-sections (Fig. 1). For example, for image from Fig. 7 the following coordinates of circles centres location of $(\mathrm{m}, \mathrm{n})$ pair and their radius $r$ were obtained: Tab. 1 . 


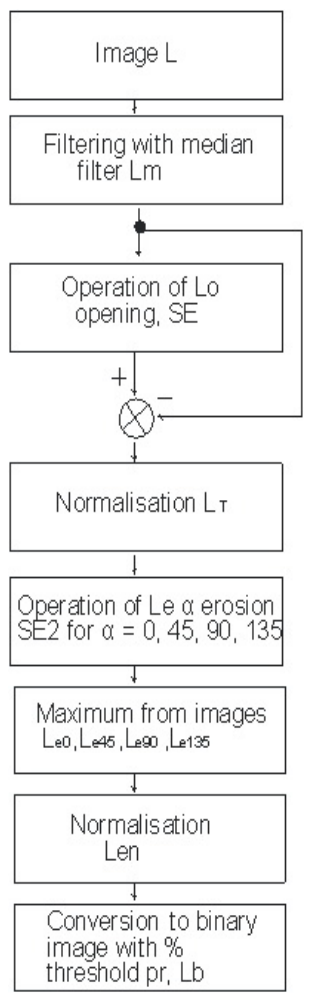

Fig. 5. Block diagram of suggested segmentation algorithm

When analysing results obtained from Table 1 and the location and number of circles visible in Fig.7, the following problems may be noticed:

- redundancy - too large number of circles found;

- determined circles not comprising the whole area of interest;

- determined circles comprising too large area of interest. These drawbacks have been eliminated using coefficients $w_{p}, w_{k}, w_{o}$ defined as follows:

$$
\begin{gathered}
w_{p}(i)=\frac{\sum_{n=1}^{N} \sum_{m=1}^{M} L_{w}(m, n)}{2 \pi r_{o}} \\
L_{w}(m, n)=\left\{\begin{array}{llc}
1 & \text { for } & \sum_{n=1}^{N} \sum_{m=1}^{M} L_{r}(m, n)=1 \wedge \\
& & \wedge \sum_{n=1}^{N} \sum_{m=1}^{M}\left(L_{p}(m, n) \oplus S E_{p}\right)=1
\end{array}\right.
\end{gathered}
$$




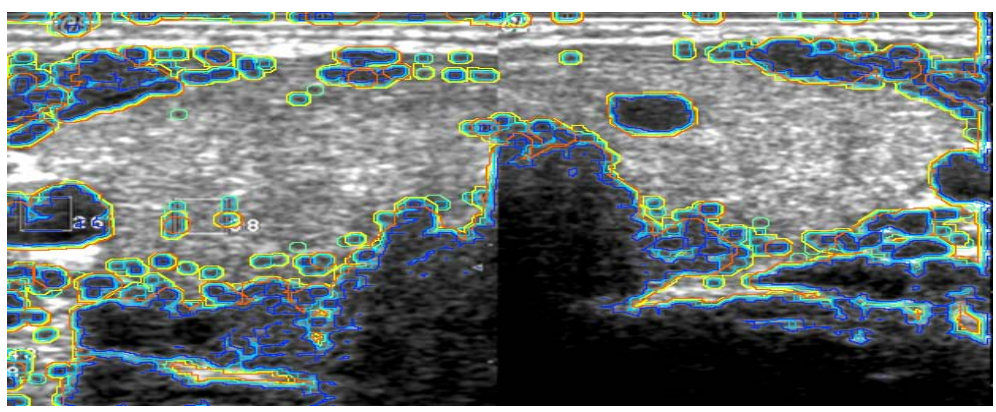

Fig. 6. Image $L_{M}$ with colour contours of resultant binary images $L b$ for $30 \%$ threshold and $S E 2$ at $k=0,45,90$, and $135^{\circ}$

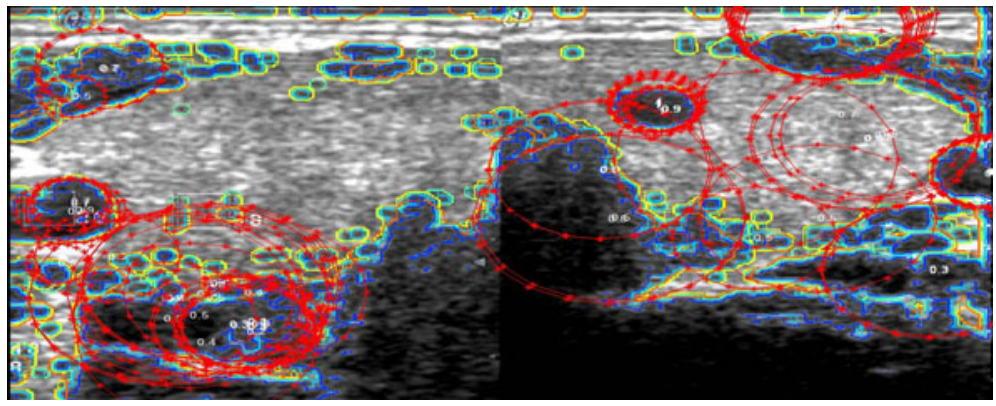

Fig. 7. Image $L_{M}$ with marked red circles determined using Hough's transform

$$
\begin{gathered}
w_{k}(i)=\frac{\sum_{n=1}^{N} \sum_{m=1}^{M}\left[L_{b}(m, n)\left(L_{r}(m, n) \bullet S E_{r}\right)\right]}{\sum_{n=1}^{N} \sum_{m=1}^{M}\left(L_{r}(m, n) \bullet S E_{r}\right)} \\
w_{o}(i)=\frac{\sum_{n=1}^{N} \sum_{m=1}^{M}\left[\left(L_{b}(m, n)\left(L_{r}(m, n) \oplus S E_{o}\right)\right)\left(1-L_{r}(m, n) \bullet S E_{r}\right)\right]}{\sum_{n=1}^{N} \sum_{m=1}^{M}\left[\left(L_{r}(m, n) \oplus S E_{o}\right)\left(1-L_{r}(m, n) \bullet S E_{r}\right)\right]}
\end{gathered}
$$

where:

$\oplus$ - Minkowski summation,

- - Minkowski closure,

$S E_{r}$ - mask of $M_{r} x N_{r}=\left(2 r_{0}+1\right) x\left(2 r_{0}+1\right)$ size, and:

$$
L_{p}(m, n)=\operatorname{xor}\left(L_{b}(m, n), L_{b}(m, n) \oplus S E\right)
$$


Individual coefficients fulfil the following role:

$w_{p}$ - is a relative measure of the number of circle points of coordinates $\left(m_{o}, n_{o}\right)$ and radius $r_{0}$ coinciding in the $S E_{p}$ mask size interval with the edge of the detected area,

$w_{k}$ - is a relative measure of percentage number of image $L_{b}$ objects points situated within the analysed circle,

$w_{o}$ - is a relative measure of percentage number of image $L_{b}$ objects points situated outside the analysed circle within the radius determined by mask $S E_{o}$.

For so defined coefficients: $w_{p}, w_{k}, w_{o}$ and having analysed individual variation in a few dozen USG images, the following values have been arbitrarily set $w_{p}>$ $0.5, w_{k}<0.2, w_{o}>0.5$. When these assumptions are satisfied $\left(w_{p}>0.5\right.$, $\left.w_{k}<0.2, w_{o}>0.5\right)$ the number of wrongly recognised circles substantially declines and only those, which are significant from the diagnostic point of view, remain (Fig. 8). For example, in Table 1 this condition is fulfilled by the circle of number $L_{p}=4$ (marked grey).

For 20 analysed patients no wrongly recognised areas have been found, which contour could be roughly approximated to a circle.

Table 1. Part of the first nine elements of the table of circles centres location coordinates and their radii and weights (sizes are given in pixels)

\begin{tabular}{|l|l|l|l||l|l|l|}
\hline$L p$ & $n_{o}$ & $m_{o}$ & $r_{o}$ & $w_{p}$ & $w_{k}$ & $w_{o}$ \\
\hline \hline 1 & 31 & 262 & 20 & 0.82 & 0.63 & 0.47 \\
\hline 2 & 37 & 209 & 33 & 0.72 & 0.18 & 0.93 \\
\hline 3 & 39 & 199 & 24 & 0.70 & 0.14 & 0.65 \\
\hline 4 & 39 & 202 & 26 & 0.66 & 0.13 & 0.74 \\
\hline 5 & 40 & 92 & 21 & 0.72 & 0.29 & 0.48 \\
\hline 6 & 42 & 208 & 32 & 0.70 & 0.21 & 0.88 \\
\hline 7 & 57 & 63 & 41 & 0.64 & 0.42 & 0.74 \\
\hline 8 & 58 & 64 & 40 & 0.64 & 0.41 & 0.74 \\
\hline 9 & 62 & 255 & 46 & 0.52 & 0.61 & 0.47 \\
\hline
\end{tabular}

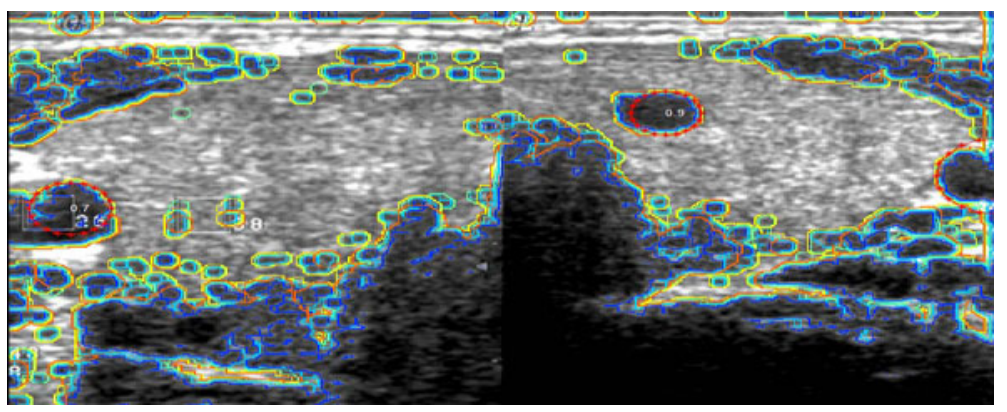

Fig. 8. Image $L_{M}$ with marked red correct (at satisfied conditions $w_{p}>0.5, w_{k}<0.2$, $\left.w_{o}>0.5\right)$ circles determined using Hough's transform 


\section{Suggested Method'S Use in Hashimoto's Disease Diagnostics}

The reference analysis areas (arteries on both sides of the trachea - areas $o_{l 1}$ and $o_{p 1}$ - Fig. 1) found, fully automatically, enable acquiring measures, interesting from diagnostic point of view, of echogenicity changes (relative grey level) in thyroid lobes limited by areas $M_{o} x N_{o}\left(o_{l 2}\right.$ and $\left.o_{p 2}\right)$. Sizes of areas $o_{l 2}$ and $o_{p 2}$, i.e. $M_{o} x N_{o}$ have been determined based on trials carried out on the test group. The best results have been obtained for $M_{o} x N_{o} \simeq 40 x 40$ pixels [2], [3] (Fig. $1)$. At anatomically regular artery the size of this area is close to its diameter $M_{o} x N_{o} \simeq s_{o} x s_{o}$ and the displacement of centres of areas $o_{l 1}$ and $o_{l 2}$ as well as $o_{p 1}$ and $o_{p 2}$ is $\simeq 2 * s o$. The obtained results of the average from differences in grey level between the areas $o_{l 1}$ and $o_{l 2}$ as well as $o_{p 1}$ and $o_{p 2}$ (Fig. 1) are presented in Fig. 9, Fig. 10, Fig. 11 for $k=10$ patients from both groups (10 healthy patients and 10 patients with Hashimoto's disease). Values of differences $o_{l p}$ in averages in areas $o_{l 1}$ and $o_{l 2}$ as well as $o_{p 1}$ and $o_{p 2}$ and of mean standard deviation $s t d_{o l}$ and $s t d_{o p}$ have been calculated from the relationship:

$$
\begin{gathered}
\Delta_{o l p}==\frac{\sum_{n=1}^{N_{o}} \sum_{m=1}^{M_{o}} o_{l 1}(m, n)+\sum_{n=1}^{N_{o}} \sum_{m=1}^{M_{o}} o_{p 1}(m, n)}{2 M_{o} N_{o}}- \\
s t d_{o l}=\sqrt{\frac{\sum_{n=1}^{N_{o}} \sum_{m=1}^{M_{o}} o_{l 2}(m, n)+\sum_{n=1}^{N_{o}} \sum_{m=1}^{M_{o}} o_{p 2}(m, n)}{2 M_{o} N_{o}}} \\
\frac{\sum_{m=1}^{N_{o}}\left(o_{l 2}(m, n)-\frac{1}{M_{o} N_{o}} \sum_{n=1}^{N_{o}} \sum_{m=1}^{M_{o}} o_{l 2}(m, n)\right)}{M_{o} N_{o}-1} \\
s t d_{o l p}=\max \left(s t d_{o l}, s t d_{o p}\right)
\end{gathered}
$$

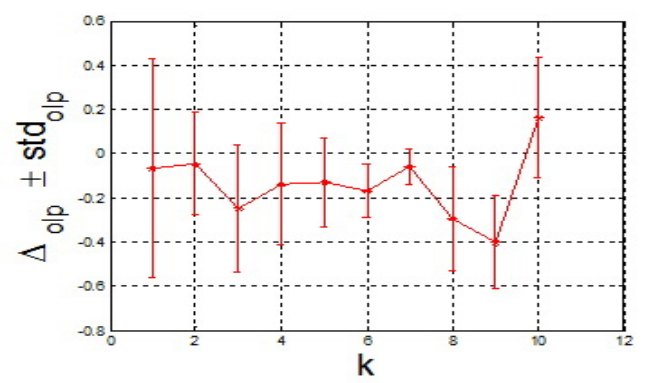

Fig. 9. Average difference between areas $o_{l 1}$ vs. $o_{l 2}$ and $o_{p 1}$ vs. $o_{p 2}$ for $k=10$ healthy patients 


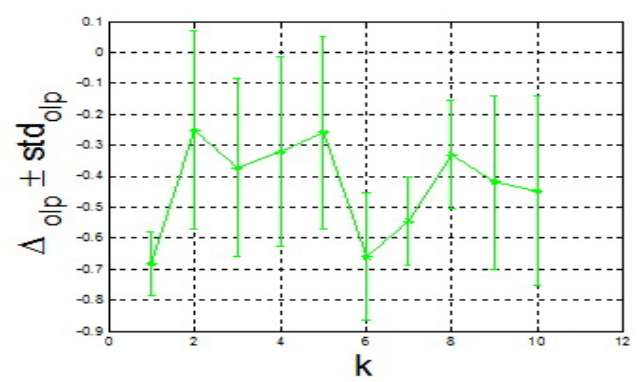

Fig. 10. Average difference between areas $o_{l 1}$ vs. $o_{l 2}$ and $o_{p 1}$ vs. $o_{p 2}$ for $k=10$ patients with Hashimoto's disease

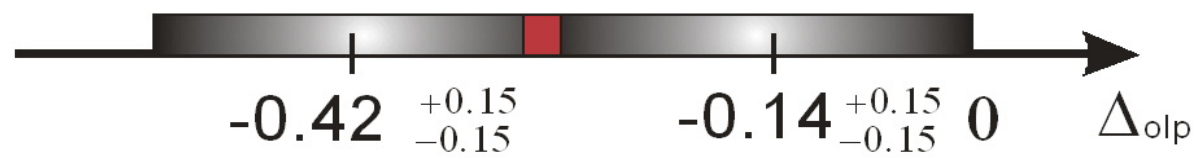

Fig. 11. Distribution on $x$ axis of differences in grey level and their standard deviation of the average for healthy patients $-0.14 \pm 0.15$ and for patients with Hashimoto's disease $-0.42 \pm 0.15$

The obtained results are shown in Fig. 9 and Fig. 10.

As it results from the results presented above, in the case of inflammation in Hashimoto disease average values of grey level difference fall within the range $\left(\Delta_{o l p} \pm s t d_{o l p}\right)-0.42 \pm 0.15$ for patients with Hashimoto's disease and $-0.14 \pm 0.15$ for healthy patients - Fig. 11. The area of uncertainty (red colour) visible in Fig. 11 , covering the range from $-0.14-0.15=-0.29$ to $-0.42+0.15=-0.27$ of the difference in pixels brightness average value is the subject of further research.

\section{Summary}

The algorithm presented in the paper enables fully automatic determination of interest areas $o_{l 1}$ and $o_{p 1}$ and based on them $o_{l 2}$ and $o_{p 2}$ enabling computation of grey level average values and other necessary statistics. In the analysis and comparison of healthy patients with patients with Hashimoto's disease it turned out that the difference in average grey levels between $o_{l 1}$ and $o_{p 1}$ and $o_{l 2}$ and $o_{p 2}$, respectively, is characteristic. In addition, it has been shown that the range of grey levels amounts to $0.42 \pm 0.15$ for patients with Hashimoto's disease and $-0.14 \pm 0.15$ for healthy patients. The algorithm presented is a fundamental algorithm for automatic finding of arteries in USG images and may be successfully used for other automatic computations of areas correlated by morphometric and/or anthropometric dimensions. 


\section{References}

1. Gutekunst, R., Hafermann, W., Mansky, T., Scriba, P.C.: Ultrasonography related to clinical and laboratory findings in lymphocyticthyroiditis. Acta Endocrinologica 121, 129-135 (1989)

2. Koprowski, R., Wrobel, Z.: Automatic segmentation of biological cell structures based on conditional opening or closing. Machine Graphics Vision 14(3), 285-308 (2005)

3. Koprowski, R., Wrobel, Z.: The automatic measurement of a staining reaction level. Machine Graphics Vision 15(2), 227-238 (2006)

4. Koprowski, R., Wróbel, Z.: Analysis of properties of automatic analysis algorithm for areas in USG image in Hashimoto's disease. Paper Submitted to ISSPA 2010 (2010)

5. Muller, H.W., Schroder, S., Schneider, C., Seiffert, G.: Sonographic tissue characterisation in thyroid gland diagnosis. A correlation between sonography and histology. Klinische Wochenschrift 63, 706-710 (1985)

6. Marcocci, C., Vitti, P., Cetani, F., Catalano, F., Concetti, R., Pinchera, A.: Thyroid ultrasonography helps to identify patients with diffuse lymphocytic thyroiditis who are prone to develop hypothyroidism. Journal of Clinical Endocrinology and Metabolism 72, 209-213 (1991)

7. Pedersen, O.M., Aardal, N.P., Larssen, T.B., Varhaug, J.E., Myking, O., VikMo, H.: The value of ultrasonography in predicting autoimmune thyroid disease. Thyroid 10, 251-259 (2000)

8. Schiemann, U., Avenhaus, W., Konturek, J.W., Gellner, R., Hengst, K., Gross, M.: Relationship of clinical features and laboratory parameters to thyroid echogenicity measured by standardized grey scale ultrasonography in patients with Hashimoto's thyroiditis. Med. Sci. Monit. 9(4) (2003)

9. Schiemann, U., Avenhaus, W., Konturek, J.W., Gellner, R., Hengst, K., Gross, M.: Standardized grey scale ultrasonography in Graves' disease: correlation to autoimmune activity. European Journal of Endocrinology 141, 332-336 (1999)

10. Szczeklik, A.: Choroby Wewnêtrzne (Internal Diseases), WNT, Kraków (2006)

11. Wrobel, Z., Koprowski, R.: Praktyka przetwarzania obrazów z zadaniami w programie Matlab (Practice of Image in the Matlab Software), Wyd. Exit, Warszawa (2004)

12. Vitti, P., Rago, T., Mancusi, F., Pallini, S., Tonacchera, M., Santini, F., Chiovato, L., Marcocci, C., Pinchera, A.: Thyroid hypoechogenic pattern at ultrasonography as a tool for predicting recurrence of hyperthyroidism after medical treatment in patients with Graves' disease. Acta Endocrinologica 126, 128-131 (1992)

13. Vitti, P., Lampis, M., Piga, M., Loviselli, A., Brogioni, S., Rago, T., Pinchera, A., Martino, E.: Diagnostic usefulness of thyroid ultrasonography in atrophic thyroiditis. Journal of Clinical Ultrasound 22 (1994) 